\title{
Relationship of the phase and amplitude parameters with anisotropy of Muller-matrix invariants
}

\author{
A. G. Ushenko, A. V. Dubolazov, V. A. Ushenko, Yu. A. \\ Ushenko, M. Yu. Sakhnovskiy, et al.
}

A. G. Ushenko, A. V. Dubolazov, V. A. Ushenko, Yu. A. Ushenko, M. Yu. Sakhnovskiy, N. Pavlyukovich, A. Bykov, A. Doronin, I. Meglinski, "Relationship of the phase and amplitude parameters with anisotropy of Muller-matrix invariants," Proc. SPIE 9970, Optics and Photonics for Information Processing X, 99701H (14 September 2016); doi: $10.1117 / 12.2237900$

SPIE Event: SPIE Optical Engineering + Applications, 2016, San Diego, California, United States 


\title{
Relationship of the phase and amplitude parameters with anisotropy of Muller-matrix invariants
}

\author{
A.G. Ushenko ${ }^{1}$, A.V. Dubolazov ${ }^{1}$, V.A.Ushenko ${ }^{1}, Y_{\text {u.A.Ushenko }}{ }^{1}$, M.Yu. Sakhnovskiy ${ }^{1}$, \\ N. Pavlyukovich ${ }^{2}$, A. Bykov ${ }^{3}$, A. Doronin ${ }^{3}$, I. Meglinski $^{3}$ \\ ${ }^{1}$ Chernivtsi National University, 2 Kotsyubinsky Str., Chernivtsi, 58012, Ukraine \\ ${ }^{2}$ Bukovinian State Medical University, Chernivtsi, 58000, Ukraine \\ ${ }^{3}$ University of Oulu, P.O. Box 4500, Oulu, Finland \\ a.dubolazov@,chnu.edu.ua
}

\begin{abstract}
A new azimuthally stable polarimetric method for processing of microscopic images of optically anisotropic structures of different biological layers histological sections is proposed. A new model of phase anisotropy definition of biological tissues by using superposition of Mueller matrices of linear birefringence and optical activity is proposed. The matrix element $M_{44}$ has been chosen as the main information parameter, which value is independent of rotation angle of both sample and probing beam polarization plane.
\end{abstract}

Keywords: polarimetry, endometrium, laser image, filtering.

\section{PART 1. THEORETICAL BASIS OF MULLER-MATRIX IMAGING}

Development of computational methodologies for processing of microscopic images is a new health-physical method. It is laser polarimetry of biological tissues histological sections [1 - 3]. It is based on measurement of the coordinate distributions (polarization maps) in the plane of polarization states of histological sections microscopic images of biological tissues. This method allows a new, inaccessible to the histological and mathematical methods of analysis, information on the optical anisotropy (linear and circular birefringence) multiscale structural elements of different biological objects.

At the same time, a complex analysis of the polarization maps of a tissue specimen is azimuthally dependent to the probing beam polarization plane and sample rotation angle. This makes it difficult to use this method in comparative research groups histological sections with different pathologies. Thus, further progress of laser polarimetry may be connected with the development of azimuthally stable methods of direct measurement of the parameters of linear and circular birefringence.

The solution of this task is using of Mueller-matrix cartography with so-called rotational invariants. It has been shown in the works $[4-6]$ that azimuthally stable, independent of the sample rotation angle $(\Theta)$ are the following matrix elements $M_{44}(\Theta)=$ const

It has been determined that in the case of optically thin (attenuation coefficient $\tau \leq 0.1$ ) layers the $M_{44}(\Theta)$ value is preferably defined by phase anisotropy mechanisms - linear and circular birefringences [7-20].

The separation of such mechanisms $\left(M_{44} \Rightarrow\left(\begin{array}{c}M_{44} \text {-linear } \\ M_{44} \text {-circular }\end{array}\right)\right.$ ) is possible with the help of spatial-frequency Fourier selection $[12,13]$.

Optics and Photonics for Information Processing X, edited by Khan M. Iftekharuddin, Abdul A. S. Awwal,

Mireya García Vázquez, Andrés Márquez, Mohammad A. Matin, Proc. of SPIE Vol. 9970,

$99701 \mathrm{H} \cdot$ (C) $2016 \mathrm{SPIE} \cdot \mathrm{CCC}$ code: $0277-786 \mathrm{X} / 16 / \$ 18 \cdot$ doi: $10.1117 / 12.2237900$

Proc. of SPIE Vol. $997099701 \mathrm{H}-1$ 
Our research is aimed at designing the experimental method of azimuthally stable Fourier's polarimetry and spatialfrequency selection of parameter distributions of linear and circular birefringence of the blood plasma donors films and patients with liver cirrhosis.

\subsection{THE THEORY OF METHOD}

The following model concepts have been assumed [1-3] by us as the basis for an analysis of the processes of modulation of laser radiation by the polycrystalline network of biological crystals with linear and circular birefringence:

- blood plasma consists of two optical anisotropic components - globulin and albumin crystals;

- the optical anisotropic albumin network is formed by large-scale (the range of the transverse dimensions $l \sim 30 \mu m \div 100 \mu m$ ) crystals with a prevalence by linear birefringence;

- $\quad$ optical anisotropy of these structures is characterized by the distribution of the coordinate matrix element, where the - direction of the optical axis, - phase shift between linearly polarized orthogonal components of the light beam amplitude;

- the optical anisotropic component of the globulin is formed by spherulitic crystals $(l \sim 5 \mu m \div 10 \mu m ; L \approx l)$ with predominantly circular birefringence $M_{44}(\theta)$, where $\theta$ - polarization plane rotation angle.

\section{2. OPTICAL REALIZATION OF SPATIAL-FREQUENCY OF FOURIER'S SELECTION OF THE ENDOMETRIUM}

Fig. 1 presents a diagram of laser Fourier polarimeter with spatial-frequency filtration [9].

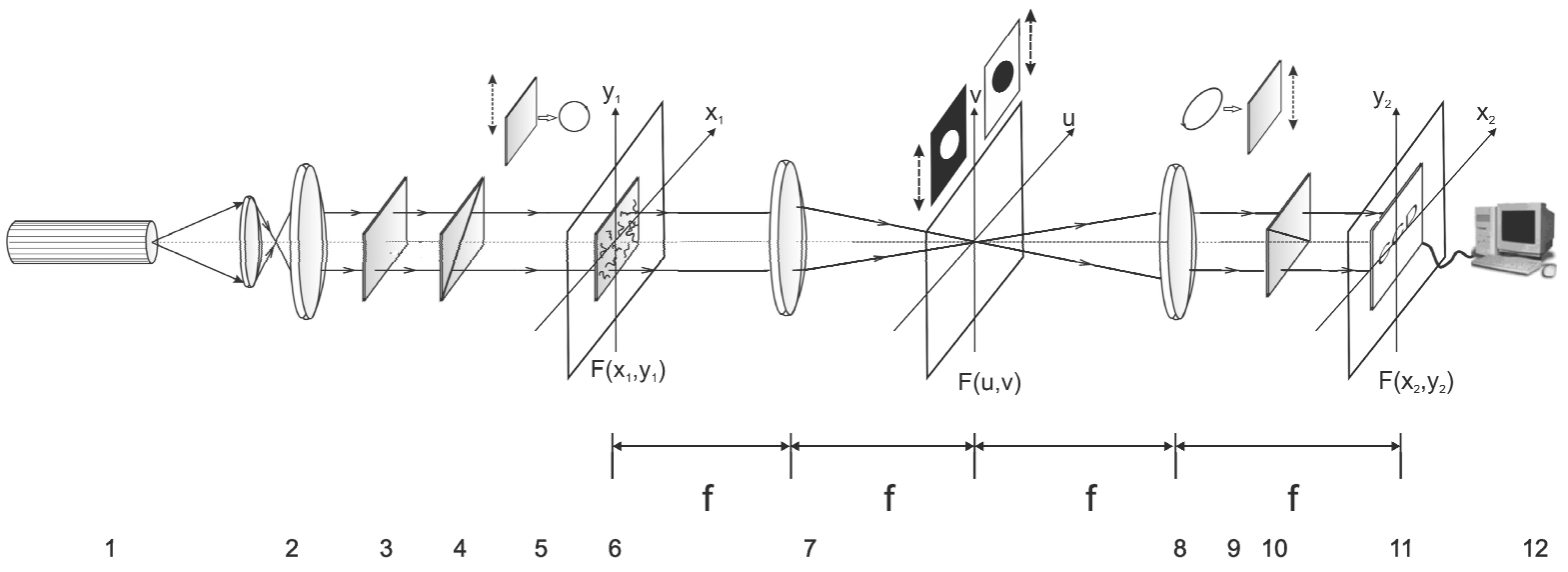

Fig. 1. Optical scheme of Fourier polarimeter, where 1 - He-Ne laser, 2 - a collimator, 3 - a stationary quarter-wave plate, 5,9 - mechanically movable quarter-wave plates, 4, 10 - a polarizer and analyzer respectively; 6 - object of study 7,8 polarization microobjectives; 11 - CCD camera; 12 - a personal computer. $F\left(x_{1}, y_{1}\right)$ - object plane, $F\left(x_{2}, y_{2}\right)-$ image plane, $F(u, v)$ - Fourier plane with the diaphragms

Illumination of a sample under study was performed by the parallel $\left(\varnothing=10^{4}\right)$ laser beam of He-Ne $(\lambda=0.6328 \mu m$, W $=5.0 \mathrm{~mW}$ ). The polarization light source consisted of quarter-wave plates 3;5 and polarizer 4, it formed a right circularly polarized beam. Blood plasma films were placed in the focal plane of polarization microobjective 7 (focal distance $-30 \mathrm{~mm}$, aperture -0.1 , magnification $-4 \mathrm{x}$ ). Behind the (Fourier) focal plane the vignetting diaphragm was located, its size changed within the range of $2 \mathrm{pix}$ to $300 \mathrm{pix}$. Polarization microobjective 8 (focal distance $-30 \mathrm{~mm}$, aperture -0.1 , magnification $-4 \mathrm{x}$ ) was located at the focal length form the frequency plane of lens 7 and, thus, performed inverse Fourier transform of a filtered out polarization field of laser radiation. The coordinate distribution of intensity of such fields, polarizationally filtered by quarter-wave plate 9 and polarizer 10, was registered in the plane of 
CCD-camera 11 (The Imaging Source DMK 41AU02.AS, monochrome 1/2" CCD, Sony ICX205AL (progressive scan); resolution - 1280x960; light sensitive area size - 7600x6200 $\mu \mathrm{m}$; sensitivity - 0.05 lx; dynamic range -8 bit; SNR - 9 bit, deviation of photosensitive characteristics from linear no more then $15 \%$ ). It provided the range of measuring the structural elements of polycrystalline network with the resolution of 2-2000 $\mathrm{mm}$ [18-20].

Matrix element $M_{44}$ was calculated for each probing beam within every pixel $(m \times n)$

$$
M_{44}=\frac{I_{\circledast}^{\otimes}-I_{\oplus}^{\otimes}}{I_{\circledast}^{\otimes}+I_{\oplus}^{\otimes}}-0.5\left(\frac{I_{\circledast}^{0}-I_{\oplus}^{0}}{I_{\circledast}^{0}+I_{\oplus}^{0}}+\frac{I_{\circledast}^{90}-I_{\oplus}^{90}}{I_{\otimes}^{90}+I_{\oplus}^{90}}\right) .
$$

Here $I_{\otimes}^{\otimes ; 0 ; 90} ; \quad I_{\oplus}^{\otimes ; 0 ; 90}$ - are the intensities of spatial-frequency filtered image $(\Theta ; \quad \oplus)$ for each polarization states of probing beam $\left(\otimes ; \quad 0^{0} ; \quad 90^{0}\right)$.

To quantify the coordinate distributions we used statistical analysis [7-10]. We calculated a set of statistical moments of the 1-st - 4-th orders $Z_{j=1 ; 2 ; 3 ; 4}$ using the following algorithms

$$
Z_{1}=\frac{1}{N} \sum_{i=1}^{N}\left|(q)_{i}\right|, Z_{2}=\sqrt{\frac{1}{N} \sum_{i=1}^{N}(q)_{i}^{2}}, Z_{3}=\frac{1}{\left(Z_{2}\right)^{3}} \frac{1}{N} \sum_{i=1}^{N}(q)_{i}^{3}, Z_{4}=\frac{1}{\left(Z_{2}\right)^{4}} \frac{1}{N} \sum_{i=1}^{N}(q)_{i}^{4}
$$

The obtained data indicate the distribution histogram of random values optically anisotropic structures in the plane of the histological section.

\section{PART 2. MULLER-MATRIX TOMOGRAMS OF BIREFRINGENCE BIOLOGICAL LAYERS}

\subsection{RESEARCH OBJECTS}

As objects of investigation, we chose two groups of optically-thin (attenuation coefficient $\tau \approx 0,087 \div 0,098$ ) single scattered endometrium donors (36 samples - group 1) and patients with pathology (36 samples - group 2).

The samples were prepared on a freezing microtome, using a standard technique. From the optical point of view such samples characterized by transformation of polarization without depolarization.

Fig. 2 shows the classic microscopic images of samples of both groups.

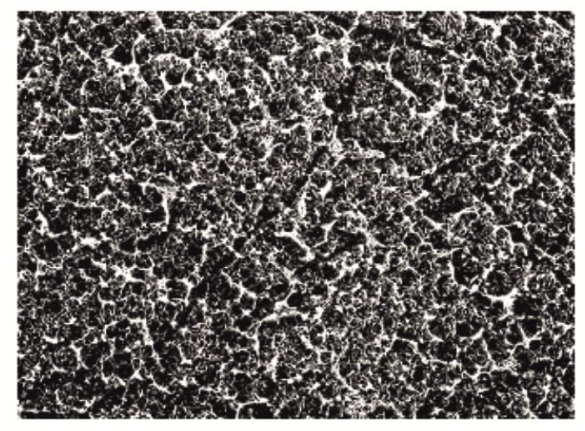

a)

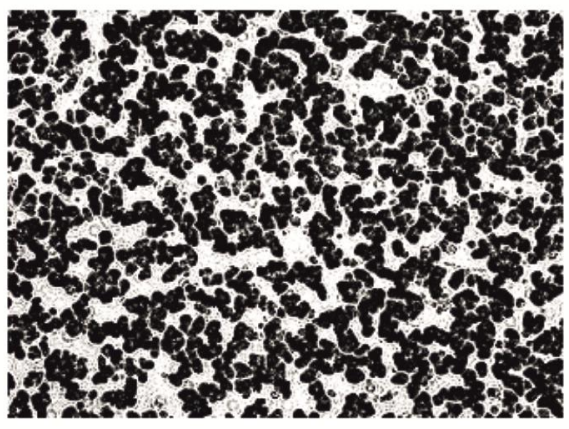

b)

Fig. 2. Microscopic images of the endometrium: group 1 (a) and group 2 (b)

As can be seen, coordinate large-scale structure of such classic microscopic images is similar. This fact makes it difficult to histological differentiation of endometrium. 


\subsection{SPATIAL-FREQUENCY FOURIER POLARIMETRY OF LINEAR BIREFRINGENCE OF THE ENDOMETRIUM}

For the purpose of choosing optimal conditions of spatial-frequency filtration the following range $\Delta r=2$ pix $\div 50$ pix of possible sizes of the vignetting diaphragm.

The criterion for diaphragm size choosing is simultaneous change of the set of statistic moments $Z_{j=1 ; 2 ; 3 ; 4}$ (4) of $M_{44}$. In our case the optimal size was $\Delta r=30 p i x$.

Such geometric size was chosen for comparative investigations of optical anisotropy of the fibrilar networks of the endometrium tissue, which characterize by coordinate distributions of rotational invariant $M_{44}(\delta)$ - Fig.3.

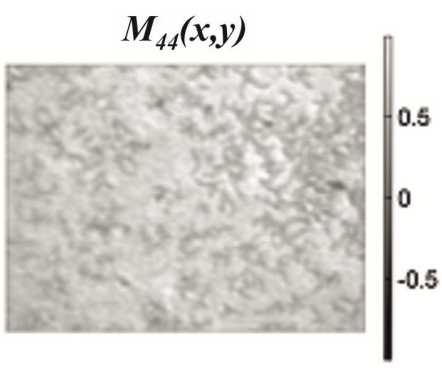

1)

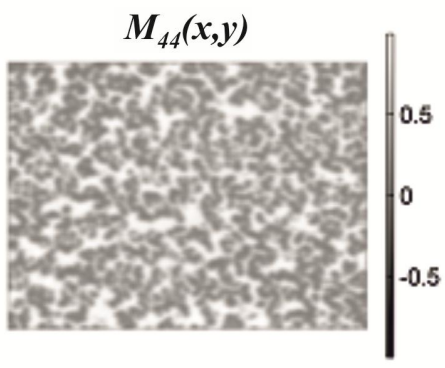

3)

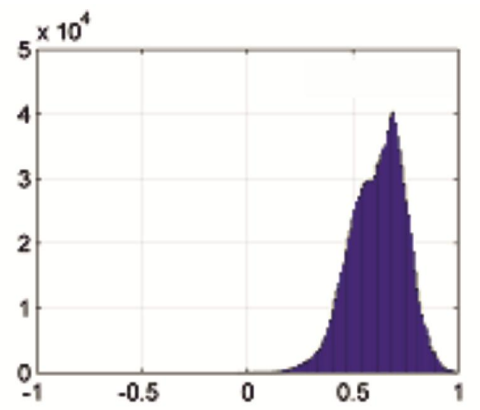

2)

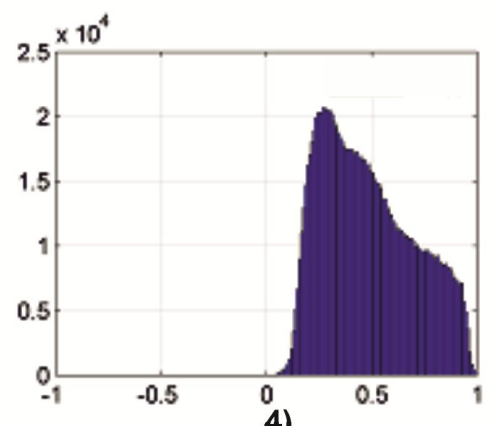

4)

Fig. 3. "Low-frequency" coordinate maps $M_{44}(\delta)(1),(3)$ histograms (2),(4) of the linear birefringence distribution of endometrium of group $1((1),(2))$ and group 2 ((3),(4)).

A comparative analysis of aggregate parameters, that characterize matrix maps $M_{44}(\delta)$ of linear birefringence of largescale optically anisotropic networks of the endometrium plasma samples, revealed some discrepancies between them. Namely, the main extrema of histograms of the distributions of random matrix maps $M_{44}(\delta)$ values for histological sections of both types are localized in different areas. Thus, the most probable is value $M_{44}(\delta) \sim 0.3$ is for a group 1 (fig. 3,(2)); for a sample $2 M_{44}(\delta) \sim 0.6$ (fig. 3,(4)). The revealed peculiarity, in our opinion, is connected with a more developed structure of the endometrium of ill patient. Quantitatively differences between matrix maps $M_{44}(\delta)$ of the protein matrices of the endometrium of both types illustrate average values and standard deviations of the set of statistic $Z_{i=1 ; 2 ; 3 ; 4}$ parameters, illustrated in the table 1 . Also, let us introduce the specificity parameter $S p=a(a+b)^{-1}(a, b-$ the number of the correct and incorrect diagnoses within group) in differentiation of normal - liver cirrhosis. 
Table 1. Parameters of the statistic structure of matrix maps $M_{44}(\delta)$ of linear birefringence of endometrium.

\begin{tabular}{|c|c|c|c|}
\hline \multirow{2}{*}{ Parameters } & \multicolumn{2}{|c|}{$M_{44}(\delta)$} & \multirow{2}{*}{$S p$} \\
\cline { 2 - 3 } & normal & pathology & \\
\hline$Z_{1}$ & $0,25 \pm 0,059$ & $0,58 \pm 0,11$ & $80 \%$ \\
\hline$Z_{2}$ & $0,10 \pm 0,022$ & $0,14 \pm 0,031$ & $60 \%$ \\
\hline$Z_{3}$ & $1,23 \pm 0,37$ & $0,75 \pm 0,14$ & $85 \%$ \\
\hline$Z_{4}$ & $1,49 \pm 0,28$ & $1,06 \pm 0,19$ & $78 \%$ \\
\hline
\end{tabular}

A comparative analysis of the data of laser-frequency Fourier's - polarimetry of linear birefringence of endometrium of both groups of patients revealed sensibility to a differentiation of such states of all statistic moments of the $1^{\text {st }}$ through the $4^{\text {th }}$ orders with specificity level $S e \sim 78 \%-83 \%$ (printed in grey color in table 2 ).

\subsection{SPATIAL-FREQUENCY FOURIER POLARIMETRY OF CIRCULAR BIREFRINGENCE OF THE ENDOMETRIUM}

Diagnostic possibilities of differentiating of pathologically changed samples of the endometrium tissue, using the method of high frequency spatial-frequency Fourier-domain - polarimetry of circular birefringence employing opaque filter illustrate probable dependences of matrix maps $M_{44}(\theta)$ adduced in a series in fig. 4.

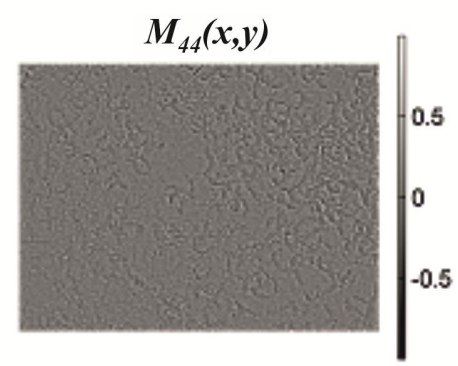

1)

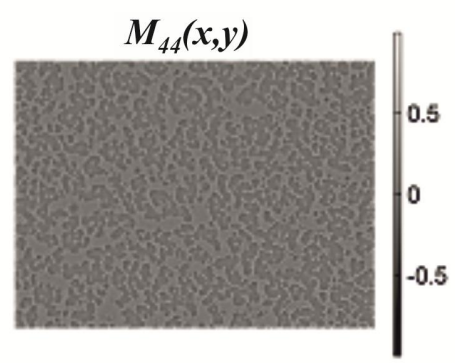

3)
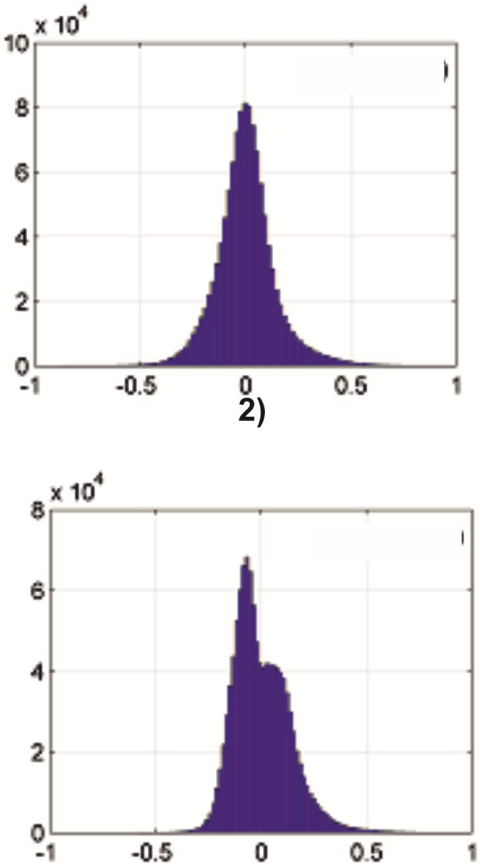

4)

Fig. 4. "High frequency" coordinate maps $M_{44}(\theta)(1),(3)$ histograms (2),(4) of the linear birefringence distribution of the endometium of group 1 ((1),(2)) and group 2 ((3),(4)).

Comparing the findings about the structure of the distributions of circular birefringence index under the conditions of high frequency filtration of laser field radiation - it is range expansion of random values change $M_{44}(\theta)$ for a histogram of the matrix map at the expense of a "pathologic" increase of the endometrium tissue. 
Quantitative differences between matrix maps $M_{44}(\theta)$ of circular birefringence of polycrystalline collagenous networks of both types illustrate average values and standard deviations of statistic set $Z_{i=1 ; 2 ; 3 ; 4}$ parameters, educed in table 2 .

Table 2. Parameters of the statistic structure of matrix maps $M_{44}(\theta)$ of circular birefringence of endometrium.

\begin{tabular}{|c|c|c|c|}
\hline \multirow{2}{*}{ Parameters } & \multicolumn{2}{|c|}{$M_{44}(\theta)$} & \multirow{2}{*}{$S p$} \\
\cline { 2 - 3 } & normal & pathology & \\
\hline$Z_{1}$ & $0,07 \pm 0,011$ & $0,10 \pm 0,017$ & $57 \%$ \\
\hline$Z_{2}$ & $0,12 \pm 0,018$ & $0,17 \pm 0,031$ & $62 \%$ \\
\hline$Z_{3}$ & $0,15 \pm 0,021$ & $0,49 \pm 0,39$ & $90 \%$ \\
\hline$Z_{4}$ & $0,34 \pm 0,043$ & $0,62 \pm 0,071$ & $80 \%$ \\
\hline
\end{tabular}

A comparative analysis revealed the following oncologic condition with a high level of specificity ( $78 \% \leq S e \leq 89 \%$ ) of uterus parameters (depicted in grey color in table 2):

- $\quad$ statistic moments of the $1^{\text {st }}$ and the $2^{\text {nd }}$ of orders $\theta(m \times n)$, differences between values is from 2 to 3,5 times;

\section{CONCLUSIONS}

A set of criteria for a polarization-phase differentiation of endometrium (normal and pathology) has been revealed and substantiated:

- statistical moments of the $1^{\text {st }}-4^{\text {th }}$ order which characterize a distribution of azimuthally stable matrix element $M_{44}(\delta)$, stipulated by linear birefringence of the endometrium;

- statistical moments of the $1^{\text {st }}-4^{\text {th }}$ orders which characterize a distribution of azimuthally stable matrix element $M_{44}(\theta)$, stipulated by circular birefringence of the endometrium.

\section{Acknowledgement}

This work was supported by the grant №0116U001446; №0116U001449; №0115U003241; №0115U003227; №0115U003235 from the Ukraine Foundation for Basic Research.

[1] Angelsky, O.V., Zenkova, C.Y., Gorsky, M.P., Gorodynśka, N.V. "Feasibility of estimating the degree of coherence of waves at the near field," Applied Optics 48 (15), 2784-2788 (2009).

[2] Angel'skiǐ, O.V., Ushenko, A.G., Arkhelyuk, A.D., Ermolenko, S.B., Burkovets, D.N. "Structure of matrices for the transformation of laser radiation by Biofractals," Quantum Electronics, 29 (12), 1074-1077 (1999).

[3] O. V. Angelsky, R. N. Besaha, A. I. Mokhun, I. I. Mokhun, M. O. Sopin, M. S. Soskin, M.V. Vasnetsov, "Singularities in vectoral fields," Proc. SPIE 3904, 40 (1999).

[4] Ushenko, Y.,A., Boychuk, T.,M., Bachynsky, V.,T., Mincer, O.,P., "Diagnostics of Structure and Physiological State of Birefringent Biological Tissues: Statistical, Correlation and Topological Approaches," Handbook of Coherent-Domain Optical Methods, Springer Science+Business Media New York, , p. 107-148, (2013).

[5] J.M. Bueno, J. Jaronski, "Spatially resolved polarization properties for in vitro corneas," Ophthal. Physiol. Opt. Vol. 21, No. 5, 384-392 (2001).

[6] Shribak, R. Oldenbourg, "Techniques for fast and sensitive measurements of two-dimensional birefringence distributions," Appl. Opt., Vol. 42, 3009-3017, 2003.

[7] M.H. Smith, "Interpreting Mueller matrix images of tissues," Proc. SPIE 4257, 82-89 (2001).

[8] Holovatsky, V.A., Makhanets, O.M., Voitsekhivska, O.M. "Oscillator strengths of electron quantum transitions in spherical Nanosystems with donor impurity in the center," Physica E: LowDimensional Systems and Nanostructures, 41 (8), 1522-1526 (2009). 
[9] Jóźwicki, R., Patorski, K., Angelsky, O.,V., Ushenko, A.,G., Burkovets, D.,N., “Automatic polarimetric system for early medical diagnosis by biotissue testing," Optica Applicata 32 (4), p.p.603-612, 2005.

[10] Goodman, J.,W., "Statistical properties of laser speckle patters. In: Laser Speckle and Related Phenomena," Ed. J.C. Dainty. - Berlin: Springer-Verlag, p.p. 9-75, (1975).

[11] Ushenko, Yu., A., Tomka, Yu., Ya., Dubolazov, A., V., "Laser diagnostics of anisotropy in birefringent networks of biological tissues in different physiological conditions," QUANTUM ELECTRON, 41 (2), p.p.170-175, (2011).

[12] Ushenko, V. A., Sidor, M. I., Marchuk, Y. F., Pashkovskaya, N. V., Andreichuk, D. R. “Azimuth-invariant mueller-matrix differentiation of the optical anisotropy of biological tissues," Optics and Spectroscopy, 117(1), 152-157 (2014).

[13] V.A. Ushenko, N.I. Zabolotna, S.V. Pavlov, D.M. Burcovets, O.Yu. Novakovska, "Mueller-matrices polarization selection of two-dimensional linear and circular birefringence images," Proc. SPIE 9066, Eleventh International Conference on Correlation Optics, 90661X (2013).

[14] Ushenko, V.A., Gorsky, M.P., "Complex degree of mutual anisotropy of linear birefringence and optical activity of biological tissues in diagnostics of prostate cancer," Optics and Spectroscopy 115(2), 290-297 (2013).

[15] Ushenko, Y. A., Gorskii, M. P., Dubolazov, A. V., Motrich, A. V., Ushenko, V. A., Sidor, M. I., "Spatialfrequency Fourier polarimetry of the complex degree of mutual anisotropy of linear and circular birefringence in the diagnostics of oncological changes in morphological structure of biological tissues," Quantum Electronics, 42(8), 727, (2012).

[16] Ushenko, V. A., "Complex degree of mutual coherence of biological liquids," In ROMOPTO International Conference on Micro-to Nano-Photonics III (pp. 88820V-88820V). International Society for Optics and Photonics. (2013).

[17] Ushenko, Y. O., Dubolazov, O. V., Karachevtsev, A. O., Gorsky, M. P., Marchuk, Y. F., "Wavelet analysis of Fourier polarized images of the human bile," Applied optics, 51(10), C133-C139, (2012).

[18] Ushenko, Y. A., Ushenko, V. A., Dubolazov, A. V., Balanetskaya, V. O., Zabolotna, N.I., "Mueller-matrix diagnostics of optical properties of polycrystalline networks of human blood plasma," Optics and Spectroscopy, 112(6), 884-892, (2012).

[19] Yu A Ushenko, Yu Ya Tomka, A V Dubolazov, "Laser diagnostics of anisotropy in birefringent networks of biological tissues in different physiological conditions," QUANTUM ELECTRON, 41 (2), 170-175, (2011).

[20] Ushenko, Y. A., Dubolazov, A. V., Balanetskaya, V. O., Karachevtsev, A. O., Ushenko, V. A., "Waveletanalysis of polarization maps of human blood plasma," Optics and Spectroscopy, 113(3), 332-343, (2012). 